\title{
Defect detection in mural painting by random infrared thermography associated with singular value decomposition and higher orders statistics
}

\author{
J.-L. Bodnar*, V. Vrabie**, E. Perrin**, K. Mouhoubi*, V. Detalle *** \\ *GRESPI/ECATHERM, URCA, BP 1039, 51687 Reims, France \\ **CRESTIC, URCA, Chaussée du Port, B.P. 541, 51012 Châlons-en-Champagne, France \\ *** LRMH, 29 avenue de Paris, 77420 Champs sur Marne, France
}

\begin{abstract}
In this paper, we studied the association of random infrared thermography, Singular Value Decomposition (SVD) and Higher Order Statistics (HOS) to help to restore works of art. We present first, the principle of theses signal processing methods. We show then, that this association allows detection of inclusions of plastazote located in an academic fresco. We show in a third stage that this photothermal method permits the location of defect located in a real work of art. It is the painting on canvas entitled "Saint Martin" located in the church of "Bonnet". Finally, we show that this association permits in one hand the use of a lower power density. On the other hand, it permits to reduce the influence of radiative properties of the pictorial layer and of the inhomogeneous energy deposition on the photothermal signal.
\end{abstract}

\section{Introduction}

Stimulated infrared thermography is a classical method of non-destructive testing. Concerning the restoration of works of art, it has already been used, for example, for the detection of delamination in mural paintings [1-11]. The excitation classically implemented is the pulsed one. It is a robust and a well known. Its main disadvantage is the use of an excitation close to a Dirac function. It uses then a high density of energy. It is a handicap for the analysis of fragile materials such as works of art. A possible alternative is the implementation of a random excitation. Indeed, it allows the non destructive testing of materials, using a low density excitation. It has already been implemented successfully for the analysis of work art. It was then associated with correlation or parametric analysis [12-13]. In this paper, we propose to study the association of the random infrared thermography with other modes of post-treatment. It is Singular-Value Decomposition (SVD) and Higher-Order Statistics (HOS) [14-17]. It is the results then obtained that we present here. Our presentation is divided in four parts: We first present the principle of Singular Value Decomposition and Higher-Order Statistics methods. We show then that this association allows detection of inclusions of plastazote located in an academic fresco. We show in a third part, that this association permits the location of defect located in a real work of art. It is the painting on canvas entitled "Saint Martin" located in the church of "Bonnet". Finally, we show that this association permits in one hand the use of a lower power density. On the other hand, it permits to reduce the influence of radiative properties of the pictorial layer and of the inhomogeneous energy deposition on the photothermal signal.

\section{Principle of the signal processing methods used}

\subsection{Singular Value Decomposition}


The Singular Value Decomposition (SVD) is a classical tool of signal processing. It is very powerful. It is used to decompose a signal in the most adapted orthogonal basis associated with the experimental device used. It permits to build an empirical model of this experimental device. It is then a more natural method than the classical Fourier or Laplace transforms. It may be introduced in the field of non-destructive testing. It explains this study.

Its principle is the following: First it consists to build a rectangular observation matrix (X), from a thermographic motion. It contains $m$ rows and $n$ columns $(m>n)$. A column corresponds to the set of values of all the pixels of a thermogram (thermogram is representated by a column vector). The $\mathrm{m}$ parameter is equal to the number of pixels of one image. The $\mathrm{n}$ rows parameter is a time-dependent parameter. It corresponds to the number of thermograms acquired with time.

When the observation $\mathrm{X}$ is built, we proceed to its singular values decomposition. We then use the following formula:

$$
\mathrm{X}=\mathrm{U} \cdot \Sigma \cdot \mathrm{V}^{\mathrm{T}}
$$

In this expression, $\mathrm{U}$ is a square matrix. It contains $\mathrm{m}$ rows and $\mathrm{m}$ columns. Each column is orthogonal to the others. The columns are arranged with the descendant space energy variations. They represent the main axes of the orthogonal empirical basis of the experience. They are named Empirical Orthogonal Function (EOF). Thus the first column of the matrix $U$ is named $\mathrm{EOF}_{1}$. It corresponds to the principal energy variation of the base of the empirical model of the experimentation. Then, the second column of the matrix $\mathrm{U}$ is named $\mathrm{EOF}_{2}$. It corresponds to the orthogonal direction with just less energy importance than the previous. The reasoning continues in a logical way until the $\mathrm{EOF}_{\mathrm{m}}$ column.

$\Sigma$ is a rectangular diagonal matrix. It contains $m$ rows and $n$ columns. The set of values of the diagonal corresponds to the representativeness of the previous Empirical Orthogonal Function (EOF). They are classified from the most powerful EOF to the less powerful EOF. Thus the first value $\Sigma_{1}$ of the $\Sigma$ matrix corresponds to the energy representation of the first Empirical Orthogonal Function $\mathrm{EOF}_{1}$. By similarly the second value $\left(\Sigma_{2}<\Sigma_{1}\right)$ of the $\Sigma$ matrix, corresponds to the energy representation of the second Empirical Orthogonal Function $\mathrm{EOF}_{2}$. In the same way, the reasoning continues in a logical manner until the value $\Sigma_{\mathrm{m}}$.

$\mathrm{V}$ is a square matrix. It contains $\mathrm{n}$ rows and $\mathrm{n}$ columns. Each column is orthogonal to the others. The columns are aranged with the descendant temporal energy variations. This representation is different to classical physical representation of the experimental results. It is not a classical thermogram. It is then more difficult to interpret. It explains why, we do not consider it in this first study. However, it would be interest to use it for the characterization of defect depth.

In this study, we used the properties of the singular value decomposition (SVD) in two different ways: First, we use the SVD to reduce the effects on the photothermal signal, of the inhomogeneity of energy deposition. This inhomogeneity is due first to different optical absorptivities of the pictorial layer. It is due then to an imperfect experimental device. We consider that theses influences have a predominant level of energy. In all cases, we consider that they are higher than those due to the presence of defects. Thus, to study the photothermal signatures related to defects, we propose to remove the first most powerful EOF, and to work on following ones (within the limits of those having a good signal / noise ratio).

The second goal of the SVD decomposition is to eliminate the transient part of the photothermal signal. Indeed, this elimination permits a statistical High Order analysis. In this case, we assume that this influence is also contained in the first EOF. We have then to work with the following ones. 


\section{2) Higher Order Statistics}

Higher-Order Statistics are conventional signal processing tools. They are classically used to separate independent energy sources. They are also used to estimate the distance with the gaussianity of a random variable. In this work, we used this last property. Indeed, photothermal default signature has no reason to have a Gaussian density function. It has no reason to be statistically perfect. It will be therefore detectable by Higher Order Statistics. In this work, we considered the $3^{\text {rd }}$ and $4^{\text {th }}$ orders statistics. The $3^{\text {rd }}$ high order statistic is called "Skweness". It measures the asymmetry of the probability density function of a random variable (2)

$$
\text { Skewness }=\frac{n}{(n-1)(n-2)} \sum_{i=1}^{n}\left(\frac{X_{i}-\bar{X}}{\sigma}\right)^{3}
$$

In the case of restoration of works of art by stimulated infrared thermography, this asymmetry still exists. It is by nature linked to the non-Gaussian aspect of the thermal diffusion phenomena. However, with the presence of a default, this asymmetry should be modified. The calculation of "skewness" parameter for each pixel of the thermograms should allow the detection of the defect.

The $4^{\text {th }}$ high order statistic is called "Kurtosis." It is the flattening or the peakedness estimation of the distribution of a random variable (3)

$$
\text { Kurtosis }=\frac{n(n+1)}{(n-1)(n-2)(n-3)} \sum_{i=1}^{n}\left(\frac{X_{i}-\bar{X}}{\sigma^{4}}\right)^{4}-3 \frac{(n-1)^{2}}{(n-2)(n-3)}
$$

This dispersion depends on thermophysical properties of the studied material. However the presence of a defect changes locally these thermophysical properties. It should therefore induce a change in the Kurtsosis values. It should then permit the detection of the defect.

\section{3) The experimental device used}

The experimental systems implemented for the study is the SAMMTHIR system. It was developed in the GRESPI laboratory of the Reims University. It is composed of 3 mains systems. The first one is an optical excitation device. It is composed of a couple of halogen lamps. The second one is the optical detection chain. It is composed of an infra-red camera of thermography. For our study, we have chosen an A20 FLIR Systems infra-red camera. Finally, the experimental system implemented for this study used electronic control and data processing software to allow photothermal analysis (figures 1).

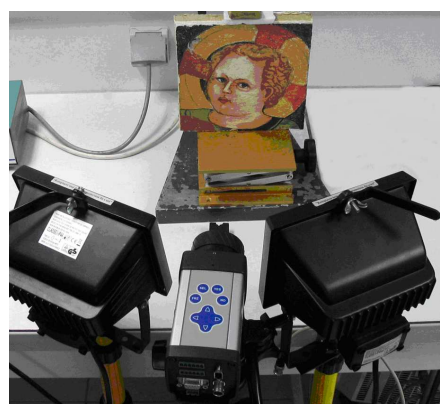

Figure 1: The experimental device used 


\section{4) Examples of obtained results}

\section{1) Analyze of an academic fresco}

The first sample studied is an academic fresco (figure 2). It was realized according to the technique of the Italian primitives. A substrate of plaster was covered with a mixture of lime and plaster. The whole was then covered, "al fresco" with a pictorial layer. It represents the child Jesus. It is a partial copy of the mural painting entitled "Saint Christophe" of the "Campana" collection in the "Louvre". To study the possibilities of the photothermal method for defects detection, Gabriela Szatanick, the professional conservator who has carried out this fresco, has introduced five inclusions of plastazote (materials whose thermal properties are close to those of the air). The first defect is located in the upper and left part of the fresco. Its depth is equal to $3 \mathrm{~mm}$. The second defect is located in the upper and right part of the fresco. Its depth is equal to $5 \mathrm{~mm}$. The third defect is located in the lower and left part of the fresco. Its depth is equal to $3 \mathrm{~mm}$. The fourth defect is located in the lower and right part of the fresco. Its depth varies between $3 \mathrm{~mm}$ and $10 \mathrm{~mm}$. The last defect is located in the upper and left part of the fresco too. Its depth is equal to $10 \mathrm{~mm}$. It is hidden by the first defect (figure $2 b$ ).

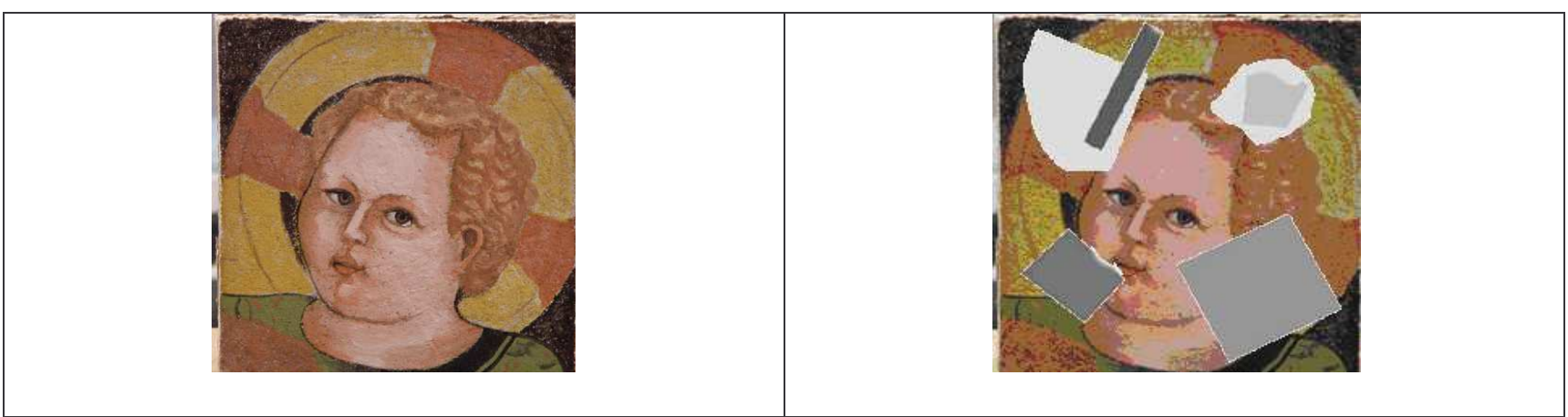

Figure 2: the studied academic fresco (a), the position of internal defects (b)

The experimental conditions selected are the followings. The excitation sequence is a pseudo random binary type. The length of the sequence is equal to 256 terms. The sample frequency is equal to $1 \mathrm{~Hz}$. The power of excitation is equal to $2 * 250 \mathrm{~W}$. In figure 3 , we present three examples of obtained results. Figure 3a corresponds to the photothermal response of the studied fresco, 240s after the beginning of the excitation. It shows specific photothermal signatures located at the place of the defects. The photothermal method is then usable to defects detection in mural paintings. The figure 3a shows then a more important photothermal signature in the right side of the thermogram. It is due to a more important deposit of energy on the right of the work of art. It is a disadvantage. It can induce artifacts of detection. To eliminate this disadvantage, we have process to a SVD of the photothermal motion. In Figure $3 \mathrm{~b}$ we present the results obtained with the calculation of the fourth Empirical Orthogonal Function (EOF4). It shows, as provided in the theory, that the SVD treatment seems to reduce the influence of an imperfect energy deposition. Thus disruptive photothermal signature located on the right of the raw image is less visible after SVD decomposition. Figure 3c corresponds to the "Skewness" calculation. It merges in a synthetic thermogram the informations contained in the EOF of order higher than 2 (and lower than those too noisy). We consider in this example, an analyze windows presenting a width equal to $100 \mathrm{~s}$. Figure $3 \mathrm{c}$ shows clearly 4 photothermal signatures located at the position of the defect. It shows then that the signatures of the less deep defects are more marked than those corresponding to deeper defects. It seems to open the 
way to the characterization of these defects. Finally, it shows that the influence of the pictorial layer and the inhomogeneity of deposition of energy have been reduced. It is a very interesting result!

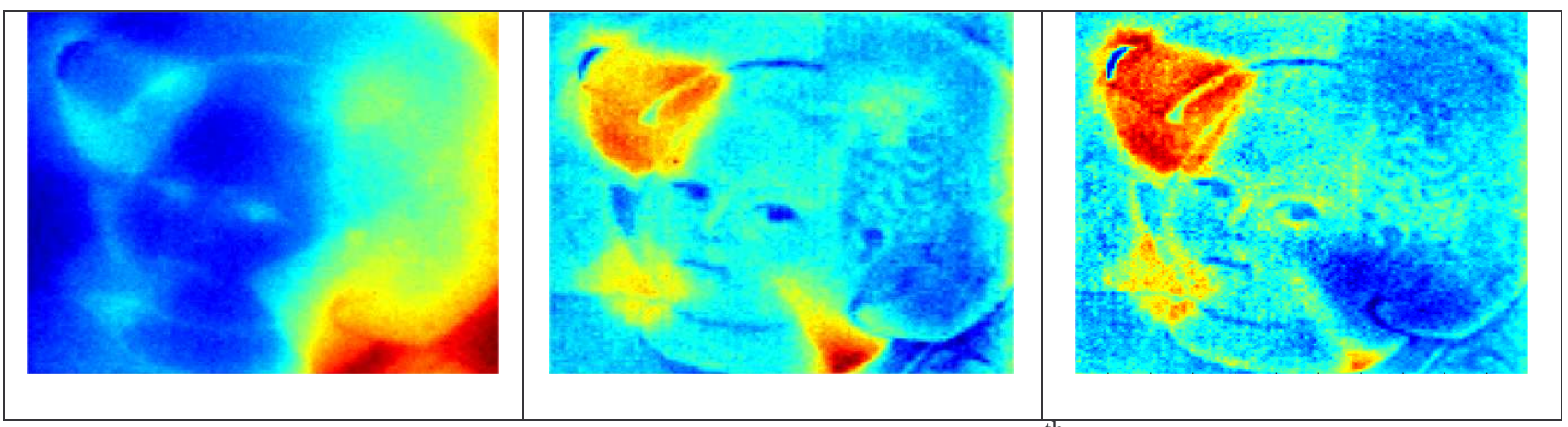

Figure 3: Photothermal response obtained after 240s, the $4^{\text {th }}$ EOF, the Skewness obtained

4.2) Analyze of the painting on canvas « Saint Martin» of the church of « Bonnet»

Following this first analysis, rather positive, but developed in laboratory, we developed an in situ analysis of a real work of art. It is a painting on canvas. It dates from the 19th century. It is located in the apse of the church of "Bonnet". It represents "Saint Martin" (Figure 4).

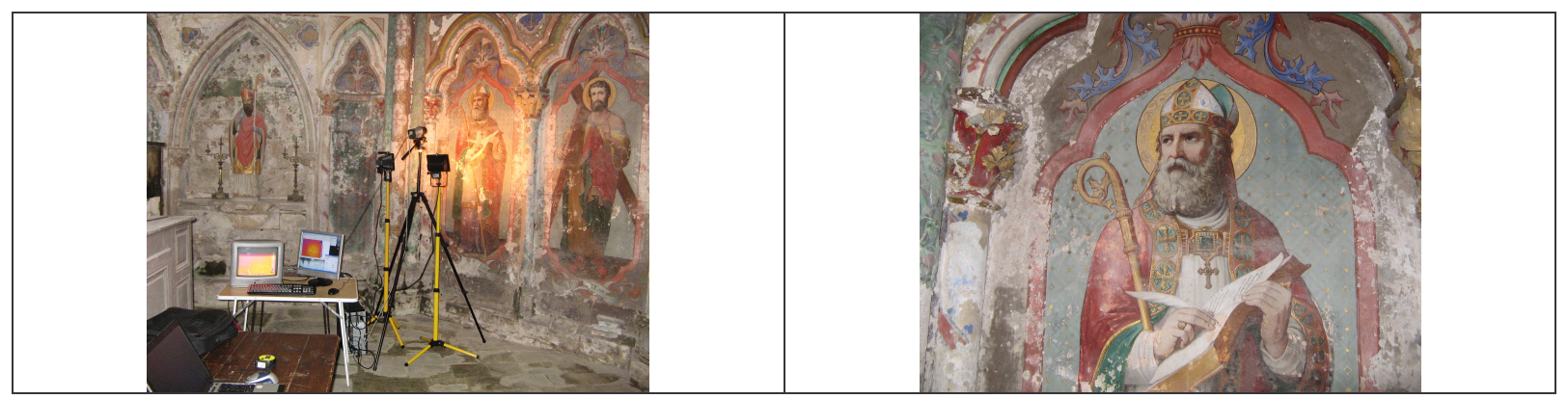

Figure 4: Painting on canvas representing « Saint Martin » of the church of Bonnet

We analyzed first this painting on canvas in an acoustic way. The results obtained makes appear a multitude of "hollow" tones, distributed rather in periphery of the work of art (Figure 5).

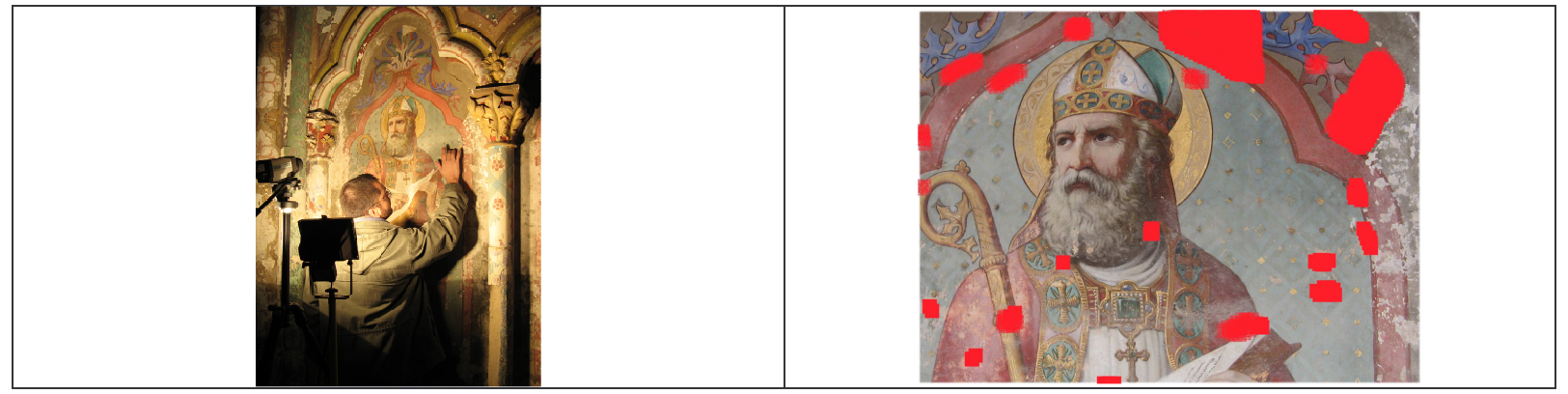

Figure 5: Acoustic analysis of painting on canvas representing « Saint Martin » of the church of Bonnet

We then analyzed this painting canvas by random infrared thermography. The experimental conditions selected are the followings. The excitation sequence is a pseudo random binary type. The length of the sequence is equal to 256 terms. The period of analyze is equal to $15 \mathrm{~s}$. The power of excitation is equal to $2 * 500 \mathrm{~W}$. In figure 6 , we present two types of photothermal results. The 
figure 6a corresponds to an ARMA parametric analysis [12-13]. The figure $6 \mathrm{~b}$ corresponds to a "Kurtosis" analysis. They show both the possibilities of the photothermal method for defect detection. Indeed they provide similar results to those obtained by acoustic analysis. They show also, as theoretically expected, that the HOS method seems less sensitive to the pictorial layer than the ARMA method. This is an advantage for the detection of defects. It can remove artifacts of detection. It is also a disadvantage. It does not allow the positioning of the defect compared to the pictorial layer.

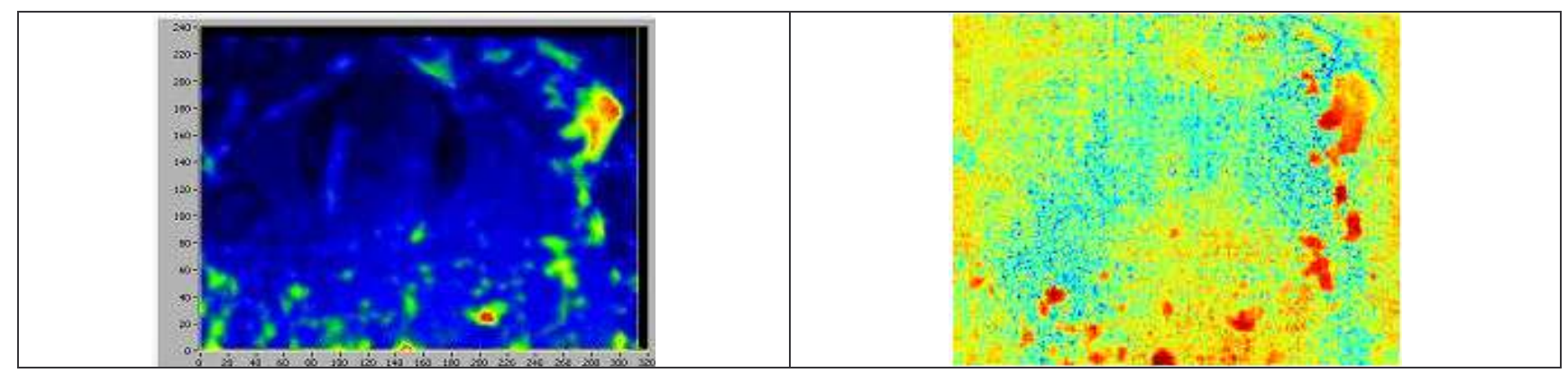

Figure 6: The ARMA and « kurtosis » results obtained

\section{5) Conclusion}

In this paper, we approached the possibilities of random infrared thermography associated with singular value decomposition and higher order statistics for the restoration of work of art.

We presented first the principle of these two signal processing methods.

We showed in a second stage, that random infrared thermography associated with SVD treatment allowed the detection of inclusion of plastazote located in an academic fresco.

We underlined then that this association seems to reduce the influence of the inhomogeneous energy deposition on the photothermal signal. It is a very interesting result. Indeed, this association seems to reduce the artifacts of detection and therefore facilitate the location of defects. We underlined in addition, the use of a random excitation. It is a technique using a low density of excitation. It leads then to a low increase of the temperature of the studied sample. It is an advantage for analyzing of fragile samples like work of art. We underlined finally, that this association leads to photothermal signatures varying with the depth of the defect. It seems then to permits a dimensional characterization of these defects.

Finally, we show that this photothermal method permit the detection of defects located in a real painting on canvas entitled "Saint Martin" of the Church of Bonnet. We underlined then, that a "Kurtosis" analysis seems to reduce the influence of an inhomogeneity of energy deposition on the photothermal response too. It is an advantage for the defects detection. Indeed it seems to reduce the artifacts of detection. It is also a disadvantage. It does not allow the positioning of the defect compared to the pictorial layer.

The results presented in this study were obtained with particulars samples. They have now to be generalized and extended. It would be interesting to study the possibilities of the association for the defect dimensional characterization. Studies going in this direction are in progress. 


\section{References}

[1] G. Szatanik : Etude et restauration d'une peinture murale représentant Saint Christophe (collection Campana, Louvre). Essai d'application de la thermographie infrarouge stimulée pour l'examen du support. INP, sept 2004

[2] J.C. Candoré : Détection et caractérisation de défauts par thermographie infrarouge stimulée : Application au contrôle d'œuvres d'art. Phd thesis, Reims, jui 2010.

[3] E. Grinzato, P.G. Bison, S. Marinetti, V. Vavilov, : Non destructive evaluation of delamination in fresco plaster using transient infrared thermography. Research in NDE, vol 5, $\mathrm{n}^{\circ}$ 4, pp257-274, 1994.

[4] G. Schirripa Spagnolo, G. Guattari, E. Grinzato : Frescoes Diagnostics by electro-optic holography and infrared thermography. Proc I the 6th World Conference on NDT and Microanalysis in Diagnostics and Conservation of Cultural and Environnemental Heritage, Rome, 1999, p. 385-398.

[5] E. Grinzato, P.G. Bison, S. Marinetti: Monitoring of ancient buildings by the thermal method. Journal of Cultural Heritage 3 (2002) 21-29.

[6] E. Grinzato, C. Bressan, S. Marinetti, P. Bison, C. Bonacina: Monitoring of the Scrovegni chapel by IR thermography: Giotto at infrared, Infrared physics and technology, vol 43, $n^{\circ} 3-5$, 2002, pp165-169.

[7] G. Carlomagno, C. Meola: Comparison between thermographic techniques for frescoes NDT, NDT\&E International 35 (2002) 559-565.

[8] N. Avdelidis, A. Moropoulou : Applications of infrared thermography for the investigation of historic structures, Journal of Cultural Heritage 5 (2004) 119-127.

[9] G. Szatanik, M. Gerard, J.L.Bodnar : La thermographie infrarouge stimulée pour l'examen des enduits dans les peintures murales : Application au Saint Christophe portant l'enfant Jésus. Journal de Conservation et restauration (coré), $\mathrm{n}^{\circ} 17$, décembre 2006, pp $64-70$.

[10] J. C. Candoré, J.L Bodnar, V. Detalle, P. Grossel : Non destructive testing of work of art by stimulated infrared thermography. European physical journal Applied Physics, 2012, vol 57, 02, pp $21002-210011$.

[11] J.C. Candoré, J.L Bodnar, V. Detalle, P. Grossel : Characterization of defects situated in a fresco by stimulated infrared thermography, European physical journal Applied Physics, 2012, vol 57, 01, pp $11002-11008$.

[12] J.L Bodnar, J.L. Battaglia : Thermophysical parameters estimation using the system identification approach. European workshops METTI 2005, 17-21 jan 2005.

[13] J.L. Bodnar, J.C. Candoré, J.N. Nicolas, D. Caron and P. Grossel: Random photothermal thermography: Principle and examples of applications, Proc QIRT 2010, pp 391-398.

[14] V. Vrabie, Statistiques d'ordre supérieur : application en géophysique et électrotechnique, thèse de doctorat de l'Institut National Polytechnique de Grenoble, 2003.

[15] V. Vrabie, Statistiques d'ordre supérieur. EUE, ISBN 6131537232, 2010.

[16] A. Khan, V. Vrabie, J.I. Mars, A. Girard, G. D’Urso, A source separation technique for processing of thermometric data from fiber optic DTS measurements for water leakage identification in dikes, IEEE Sensors Journal 2008; 8(7): 1118-1129.

[17] A.A. Khan, V. Vrabie, J. Mars, A. Girard, G. D’Urso: Automatic monitoring system for singularities detection in dikes by DTS data measurement, IEEE Trans. on Instrumentation and Measurement 2010; 59(8):2167-2175. 